\title{
Profiling the $\mathrm{N}$-Glycan Composition of IgG with Lectins and Capillary Nanogel Electrophoresis
}

\author{
Grace $\mathrm{Lu}$ and Lisa A. Holland*๑ \\ C. Eugene Bennett Department of Chemistry, West Virginia University, Morgantown, West Virginia 26506, United States
}

Supporting Information

ABSTRACT: Glycosylated human IgG contains fucosylated biantennary $\mathrm{N}$ glycans with different modifications including $N$-acetylglucosamine, which bisects the mannose core. Although only a limited number of IgG $N$-glycan structures are possible, human IgG $N$-glycans are predominantly biantennary and fucosylated and contain varying levels of $\alpha 2-6$-linked sialic acid, galactose, and bisected $\mathrm{N}$ acetylglucosamine. Monitoring the relative abundance of bisecting $\mathrm{N}$-acetylglucosamine is relevant to physiological processes. A rapid, inexpensive, and automated

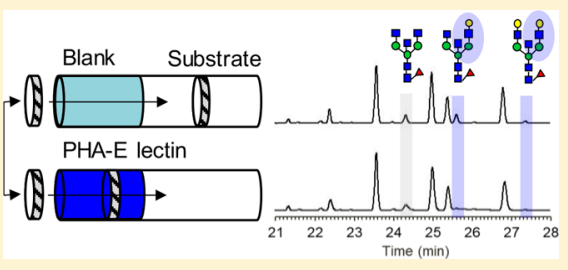
method is used to successfully profile N-linked IgG glycans and is suitable to distinguish differences in bisection, galactosylation, and sialylation in $\mathrm{N}$-glycans derived from different sources of human IgG. The separation is facilitated with self-assembled nanogels that also contain a single stationary zone of lectin. When the lectin specificity matches the $\mathrm{N}$-glycan, the peak disappears from the electropherogram, identifying the $\mathrm{N}$-glycan structure. The nanogel electrophoresis generates separation efficiencies of 500000 plates and resolves the positional isomers of monogalactosylated biantennary $\mathrm{N}$-glycan and the monogalactosylated bisected $\mathrm{N}$-glycan. Aleuria aurantia lectin, Erythrina cristagalli lectin (ECL), Sambucus nigra lectin, and Phaseolus vulgaris Erythroagglutinin (PHA-E) are used to identify fucose, galactose, $\alpha 2-6$-linked sialic acid, and bisected $\mathrm{N}$-acetylglucosamine, respectively. Although PHA-E lectin has a strong binding affinity for bisected $N$-glycans that also contain a terminal galactose on the $\alpha 1-6$-linked mannose branch, this lectin has lower affinity for $\mathrm{N}$-glycans containing terminal galactose and for agalactosylated bisected biantennary $\mathrm{N}$-glycans. The lower affinity to these motifs is observed in the electropherograms as a change in peak width, which when used in conjunction with the results from the ECL lectin authenticates the composition of the agalactosylated bisected biantennary $\mathrm{N}$-glycan. For runs performed at $17{ }^{\circ} \mathrm{C}$, the precision in migration time and peak area was less than or equal to 0.08 and $4 \%$ relative standard deviation, respectively. The method is compatible with electrokinetic and hydrodynamic injections, with detection limits of 70 and 300 $\mathrm{pM}$, respectively.

G lycosylation is a post-translational modification that is important for protein stability, ${ }^{1}$ signaling, and cell-cell communications. ${ }^{2}$ Thus, $N$-glycans have significance as biomarkers for diseases such as cancer. ${ }^{3,4}$ For example, bisected $\mathrm{N}$-glycans, which contain an additional $\mathrm{N}$-acetylglucosamine, are a potential biomarker for several different types of cancers ${ }^{5-7}$ and other diseases. ${ }^{8-10}$ Analysis of the $N$-glycans on IgG is of particular interest, because antibody glycosylation is associated with the interaction between antibody and the $\mathrm{Fc}$ receptor. ${ }^{11,12}$ IgG $N$-glycans vary across species ${ }^{13}$ and within the human population. ${ }^{14} \mathrm{IgG} N$-glycans have been reported to contain more than 30 different $N$-glycan structures in humans. ${ }^{15}$ Bisected N-glycans have an abundance of 13 to $18 \%$ in IgG from human serum ${ }^{16,17}$ and are known to increase the antibody-dependent cell-mediated cytotoxicity. ${ }^{18,19}$ This is relevant to the efficacy of antibody-based biological therapeutics. Methods of antibody production have been developed to express antibodies with bisected glycosylation. ${ }^{20}$ The significance of bisected glycans has motivated analytical chemists to devise accessible methods to identify this $N$-glycan structure and to quantify the bisected glycosylation present in glycoproteins, such as IgG.

Lectins, which are proteins with specificity for different oligosaccharides, provide inexpensive molecular recognition of
$\mathrm{N}$-glycans in analytical methods. ${ }^{21}$ When lectins are immobilized for separations, the chromatographic performance of lectin affinity columns limits their use to fractionate or enrich complex samples. Alternatively, arrays can be created, in which lectins are printed in discrete regions of a chip. Lectin arrays are well-suited for high-throughput screening of glycoproteins, which contain different combinations of $\mathrm{N}$-glycans and $\mathrm{O}$ glycans. Arrays are generally used as a tool to complement other technologies because of the limitations of lectins. Lectins are subject to cross-reactivity with other $N$-glycans and there is a limited set of glycan structures recognized by lectins. Arrays are commercially available and have been applied to therapeutic antibodies. ${ }^{22}$ While arrays may also be applied to complex mixtures, analyses of mixtures of $N$-glycans are simpler to interpret if the sample is fractionated prior to identifying the glycan structure with the immobilized lectin. This requires the use of an orthogonal separation mechanism coupled to a platform that integrates a particular lectin.

Received: August 16, 2018

Accepted: December 9, 2018

Published: December 9, 2018 
Capillary electrophoresis and hydrophilic interaction liquid chromatography (HILIC) are both effective separation techniques to profile $\mathrm{N}$-glycans, providing separation times under $30 \mathrm{~min}$ and high-throughput analyses amenable to sialylated $\mathrm{N}$-glycans. ${ }^{23}$ When coupled to laser-induced fluorescence detection, both techniques have similar detection limits; however, capillary electrophoresis is better suited for small sample volumes, ${ }^{23}$ typically consuming nanoliter volumes of glycan per run as compared to 1 to $25 \mu \mathrm{L}$ volumes for HILIC. The use of $1.2 \mu \mathrm{L}$ injection volumes in capillary electrophoresis generates a significantly better detection limit of $360 \mathrm{fM}^{24}$ Both HILIC and capillary electrophoresis can incorporate retention indexing using glucose units (i.e., GU values), ${ }^{25-27}$ where the elution or migration of each $N$-glycan can be compared to a size ladder generating a unique retention index that can be matched to values in a database to corroborate structural assignments. The nanogel-lectin method is novel, as it fully resolves IgG $N$-glycans and distinguishes IgG $\mathrm{N}$-glycans from contaminant peaks or other interfering peaks. The nanogel-lectin method may be expanded to lower abundance IgG $\mathrm{N}$-glycan peaks as well.

Capillary electrophoresis, which is based on differences in charge-to-size ratio, is an efficient method for the analysis of $\mathrm{N}$ glycan samples derivatized with charged fluorophores such as 8-aminopyrene-1,3,6-trisulfonic acid (APTS). ${ }^{27}$ Electrophoretic methods have incorporated zones of lectins reconstituted in background electrolyte containing linear polymer. ${ }^{28-30}$ These reports rely on the mobility shift of the $N$-glycan peaks that match the specificity of the lectin. ${ }^{28-30}$ Lectin-based separations have not identified bisected $N$-glycans, because lectin selectivity to bisected $N$-glycans is limited to the commercially available lectin Phaseolus vulgaris Erythroagglutinin (PHA-E). PHA-E binds with strong affinity to bisected $N$ glycans that also contain a terminal galactose on an $\alpha 1-6$ linked mannose branch. ${ }^{31}$ A challenge to using PHA-E to confirm the presence of bisected $\mathrm{N}$-acetylglucosamine is that the PHA-E lectin binds with lower affinity to non-bisected $\mathrm{N}$ glycans containing galactose linked to $N$-acetylglucosamine. In addition, PHA-E lectin binds with lower affinity to bisected $N$ acetylglucosamine if the terminal galactose on the $\alpha 1-6$-linked mannose branch is removed.

A new approach to lectin-based analyses of the $N$-glycan structure is developed, which integrates nanogel to separate $\mathrm{N}$ glycans as well as to pattern proteins that provide a sequence or structural information in a separation capillary. A nanogel is a non-Newtonian fluid composed of phospholipids that selfassemble to form different morphologies with a temperaturedependence. The nanogel has a low viscosity at low temperature (i.e., $19{ }^{\circ} \mathrm{C}$ ) and a gel-like viscosity at temperatures of $25{ }^{\circ} \mathrm{C}$ and higher. ${ }^{32,33}$ Separations of complex samples of $\mathrm{N}$-glycans have been enhanced with the use of nanogels as a viscous additive in capillary electrophoresis. ${ }^{34}$ This temperature-dependent viscosity can also be harnessed to precisely load different protein-recognition elements into a capillary. ${ }^{35}$ Unlike other high-efficiency chromatographic approaches, when the nanogel separation is complete, the lectin zone within the capillary can be easily expelled and replaced with a lectin of different specificity. Furthermore, nanogel is compatible with proteins, in some cases extending protein activity. ${ }^{35,36}$ The integration of nanogel with enzymes to sequence $N$-glycans was previously demonstrated. ${ }^{37,38} \mathrm{An}$ enzyme specific for $\beta 1-4$ galactose was used in combination with the galactose binding Erythrina cristagalli lectin (ECL) to confirm linkage specificity. ${ }^{36}$ While this approach is successful for the determination of galactose, a barrier to the analysis of bisection is that no commercially available enzyme with specificity for bisection exists. To address this barrier, a nanogel electrophoresis separation was developed that resolved bisected biantennary $N$-glycans from those that are not bisected. Furthermore, the method was modified to utilize lectins for structural identification, including bisection.

The application of capillary nanogel electrophoresis to identify $N$-glycans was adapted to utilize several lectins for structural identification, including PHA-E, which has a range of binding affinities to bisected structures. The lower affinity of PHA-E to bisected $N$-acetylglucosamine that lacks terminal galactose was leveraged to confirm bisection of agalactosylated $\mathrm{N}$-glycans in capillary electrophoresis by observing changes in peak broadening. In addition, the cross-reactivity of PHA-E to non-bisected $\mathrm{N}$-glycans containing galactose linked to $\mathrm{N}$ acetylglucosamine, which confounds the identification of bisected $\mathrm{N}$-glycans, was obviated by comparing the response of these $N$-glycans to other lectins. Several lectins with structural specificity were commercially available, ${ }^{27}$ and this report focused on the use of PHA-E and three other lectins. Aleuria aurantia lectin (AAL), ECL, and Sambucus nigra lectin (SNA) bound to fucose, galactose, and $\alpha 2-6$-linked sialic acid, respectively. The capillary nanogel electrophoresis method consumed only nanoliter volumes of lectin per analysis. The separations were complete in $30 \mathrm{~min}$, and patterning any lectin within the separation capillary was achieved within $26 \mathrm{~min}$ using an automated instrument.

This new lectin electrophoresis method was successfully used to analyze IgG $N$-glycans and to verify the structural identity of peaks that were separated. With traditional capillary electrophoresis, two of the IgG $\mathrm{N}$-glycans could not be baseline resolved. However, with the nanogel separation, the major IgG $\mathrm{N}$-glycans were separated, and baseline resolution greater than 1.5 was achieved using a $20 \%$ preparation at an operating temperature of $17{ }^{\circ} \mathrm{C}$. A single lectin was introduced as a discrete zone in the capillary to confirm the identity. This provided confidence of the $\mathrm{N}$-glycan peak assignments to identify the structure of the biantennary $N$-glycans that were derived from IgG using only four lectins (AAL, ECL, PHA-E, and SNA) without the use of $N$-glycan standards or mass spectrometry. PHA-E lectin was successfully used in an online analysis to detect all biantennary $\mathrm{N}$-glycans that were bisected with $\mathrm{N}$-acetylglucosamine. With the structural assignment of IgG $N$-glycans confirmed, the nanogel separation was suitable to compare $\mathrm{N}$-glycans derived from different sources of IgG. The ratio of bisected to non-bisected $N$-glycans was shown to be significantly different between the two samples. This method offered a rapid, automated, and inexpensive approach to identify the structure of the human IgG $N$-glycans and to distinguish subtle variations in relative abundances from different batches.

\section{MATERIALS AND METHODS}

Preparation and Derivatization of Standards. The $\mathrm{N}$ glycans from IgG were cleaved using Rapid PNGase F following the manufacturer's two-step protocol. ${ }^{39}$ The IgG was first weighed and dissolved in deionized water to a concentration of $6.25 \mu \mathrm{g} / \mu \mathrm{L}$ of $\mathrm{H}_{2} \mathrm{O}$. The cleavage reaction was performed using the recommended ratio of reagents described for $100 \mu \mathrm{g}$ protein samples. Therefore, the reagent volume was increased in accordance with the use of $600 \mu \mathrm{g}$ of 
protein and 6 times the suggested volume (i.e., $96 \mu \mathrm{L}$ ). To isolate the cleaved $N$-glycans from protein, $360 \mu \mathrm{L}$ of ice-cold ethanol was added to the sample and centrifuged for $10 \mathrm{~min}$ $(10000 \mathrm{rpm})$. The supernatant was removed from the precipitate and dried using a Savant SpeedVac concentrator (Thermo Scientific, Waltham, MA). The cleaved $N$-glycans were reconstituted in $2.5 \mu \mathrm{L}$ of $30 \mathrm{mM}$ APTS in $20 \%$ acetic acid, to which $2.5 \mu \mathrm{L}$ of $1 \mathrm{M}$ of sodium cyanoborohydride in tetrahydrofuran was then added. The reaction was mixed and incubated at $37^{\circ} \mathrm{C}$ overnight. Maltoheptaose, which was used to estimate the limit of detection, was labeled using this procedure with the exception that the ratio of excess APTS was higher (maltoheptaose/APTS, 1:200). The bisecting $N$-glycan standards $(10 \mu \mathrm{g})$ used to confirm the identity of the bisecting $\mathrm{N}$-glycans in human IgG were labeled with $100 \mathrm{mM}$ APTS in $20 \%$ acetic acid, to which $2.5 \mu \mathrm{L}$ of $1 \mathrm{M}$ sodium cyanoborohydride in tetrahydrofuran was then added. The reaction was incubated at $37^{\circ} \mathrm{C}$ for $2 \mathrm{~h}$. A shorter incubation time was used for the bisected $\mathrm{N}$-glycan, as longer incubation times are only necessary when high labeling efficiency is desired. ${ }^{40}$ With the exception of the bisected $N$-glycan standard, the incubated reactions were treated to remove excess labeling reagent using a $1 \mathrm{~mL}$ DPA-6S stationary phase extraction tube (Sigma-Aldrich, St. Louis, MO), which is an extraction column composed of polyamide resin, as described previously. $^{37}$

Phospholipid and Lectin Plug Preparation. Phospholipids were prepared as described previously, ${ }^{37}$ where the DMPC and DHPC were weighed into the same vial first so that the final molar ratio is 0.5 or 2.5 for capillary-coating and -filling, respectively. The mixed solid was dissolved with 50 $\mathrm{mM}$ sodium phosphate so that the mass of nanogel was 5 and $20 \%$ for coating and filling the capillary, respectively. The vial contents were mixed using a vortex mixer until the solid was dissolved. The nanogel underwent three freeze-thaw cycles and was centrifuged at $10000 \mathrm{rpm}$ for $10 \mathrm{~min}$ at $4{ }^{\circ} \mathrm{C}$ and was then aliquoted and stored at $-20{ }^{\circ} \mathrm{C}$. When placed in the capillary electrophoresis instrument, the $20 \%$ nanogel was stored in the sample storage region which was set at $4{ }^{\circ} \mathrm{C}$. The $5 \%$ nanogel was positioned in the buffer tray.

The commercial lectins AAL, ECL, PHA-E, and SNA were purchased in powder form from the manufacturer and contained different sugars and salts as stabilizers. The excess sugars or salts present with different lectins were removed from the lectins using a $3 \mathrm{kDa}$ (Amicon Ultra centrifuge filter, Millipore Sigma, St. Louis, MO) or a $10 \mathrm{kDa}$ (Vivaspin centrifugal concentrator GE Healthcare, Chicago, IL) molecular weight cutoff filter. The lectins were dissolved in $100 \mathrm{mM}$ 3-( $\mathrm{N}$-morpholino)-propanesulfonic acid buffered to $\mathrm{pH} 7$ that also contained $0.1 \mathrm{mM}$ calcium chloride to maintain the lectin activity during purification. Each lectin (AAL, ECL, PHA-E, and SNA) was then reconstituted into $30 \%$ nanogel $50 \mathrm{mM}$ sodium phosphate ( $\mathrm{pH} 6$ ) to a final composition of $20 \%$ nanogel.

Capillary Electrophoresis. Online analysis of IgG $\mathrm{N}$ glycans was performed using a Sciex MDQ Plus (Sciex, Redwood City, CA) with a laser-induced fluorescence detector ( $3 \mathrm{~mW}$ solid-state laser, with $\lambda_{\mathrm{ex}}=488 \mathrm{~nm}, \lambda_{\mathrm{em}}=520 \mathrm{~nm}$ ). The instrument was maintained in a temperature controlled chamber $\left(13\right.$ to $17{ }^{\circ} \mathrm{C}$ ). A $25 \mu \mathrm{m}$ internal diameter, $360 \mu \mathrm{m}$ outer diameter fused silica capillary (Polymicro Technologies, Phoenix, AZ) was used for the separation. The total length of capillary was $62 \mathrm{~cm}$, and the length to the detection window was $52 \mathrm{~cm}$. Capillaries were flushed daily with $1 \mathrm{~N}$ sodium hydroxide, water, and methanol as previously reported. ${ }^{37}$ The capillary surface was passivated using 5\% nanogel ([DMPC]/ $[\mathrm{DHPC}]=0.5)$ with $1.25 \mathrm{mM}$ calcium chloride for $20 \mathrm{~min}$ at $172 \mathrm{kPa}$ (25 psi) followed by a forward flush of $50 \mathrm{mM}$ phosphate adjusted to $\mathrm{pH} 6$ using $1 \mathrm{~N}$ sodium hydroxide for 2 min at $172 \mathrm{kPa}(25 \mathrm{psi})$. To easily introduce nanogel into capillary, the temperature of the capillary cartridge was set at $19{ }^{\circ} \mathrm{C}$. Before each separation, a 5\% nanogel ([DMPC]/ $[\mathrm{DHPC}]=0.5)$ with $1.25 \mathrm{mM}$ calcium chloride was introduced for $10 \mathrm{~min}$ at $172(25 \mathrm{psi})$ to recoat the surface followed by a rinse with $50 \mathrm{mM}$ phosphate buffer at $\mathrm{pH} 6$ for 2 min at $172 \mathrm{kPa}(25 \mathrm{psi})$. The capillary was then filled with $20 \%$ phospholipid for $3 \mathrm{~min}$ at $172 \mathrm{kPa}(25 \mathrm{psi})$ and a preplug of background electrolyte for $10 \mathrm{~s}$ at $34 \mathrm{kPa}(5 \mathrm{psi})$. For lectin studies, the fixed lectin zone of $0.5 \mathrm{~cm}$ was introduced at 69 $\mathrm{kPa}(10 \mathrm{psi})$ for $7 \mathrm{~s}$ after the capillary was filled with $20 \%$ nanogel $([\mathrm{DMPC}] /[\mathrm{DHPC}]=2.5)$ and moved into the capillary first with nanogel $(69 \mathrm{kPa}$ for $28 \mathrm{~s})$ and then with aqueous buffer ( $34 \mathrm{kPa}$ for $10 \mathrm{~s})$ to create an injection preplug. Two flow injection analyses were conducted to determine the position of the lectin zone. The capillary was first filled with $20 \%$ nanogel followed by APTS, which was detectable using laser-induced fluorescence. The APTS was then pushed to the detection window either with nanogel at $69 \mathrm{kPa}$ or with aqueous buffer at $34 \mathrm{kPa}$. The mobilization velocities of the APTS when pushed with nanogel at $69 \mathrm{kPa}$ and with aqueous buffer at $34 \mathrm{kPa}$ were 0.066 and $0.045 \mathrm{~cm} / \mathrm{s}$, respectively. At these velocities (i.e., $0.066 \mathrm{~cm} / \mathrm{s}$ for $28 \mathrm{~s}$ and $0.045 \mathrm{~cm} / \mathrm{s}$ for 10 $\mathrm{s})$, the position of the lectin zone was estimated to be $2.3 \mathrm{~cm}$.

Following the introduction of lectin, the instrument was programmed to perform two wait steps that dipped the capillary in $20 \%$ nanogel and then in an aqueous buffer to reduce the residual lectin on the outer surface of the separation capillary. After the capillary was filled and patterned with nanogel, the temperature of the capillary cartridge was decreased to $17^{\circ} \mathrm{C}$ for sample injection and separation. The sample was either injected with voltage (10 kV for $4 \mathrm{~s})$ or pressure $(21 \mathrm{kPa}$ for $5 \mathrm{~s})$, as noted in the figure captions. The sample was prepared in $10 \mathrm{mM}$ phosphate buffer $(\mathrm{pH} \mathrm{6)}$ ) for electrokinetic injection to maintain reproducible peak areas following multiple injections. The sample was reconsittued in water for hydrodynamic injection to enable sample recovery if warranted. The injections were followed by the introduction of an aqueous $50 \mathrm{mM}$ phosphate post plug, of $3.4 \mathrm{kPa}(0.5 \mathrm{psi})$ for 5 s. For electrokinetic and hydrodynamic injections, detection limits of 70 and $300 \mathrm{pM}$, respectively, were obtained using maltoheptaose labeled with excess APTS. After the run was complete, a $172 \mathrm{kPa}$ (25 psi) background electrolyte flush for $10 \mathrm{~min}$ was applied in the reverse direction to push out any remaining protein toward the site of injection. Separations of $\mathrm{N}$-glycans were performed in the absence of lectin to demonstrate that the flushing protocol was sufficient to prevent carryover by the lectin (Figure S-1). Data collection and analyses were performed using 32 Karat Software version 10.2 (MDQ Plus). For runs obtained with electrokinetic or hydrodynamic injections, the integration parameters used to obtain area were a width of 0.5 , shoulder sensitivity of 250 , and threshold of 250 .

\section{RESULTS AND DISCUSSION}

Lectins were integrated with capillary nanogel electrophoresis for $\mathrm{N}$-glycan identification as demonstrated conceptually in 
Figure 1 . The capillary was loaded with nanogel to improve the electrophoresis separation. Lectins have structural or monomer

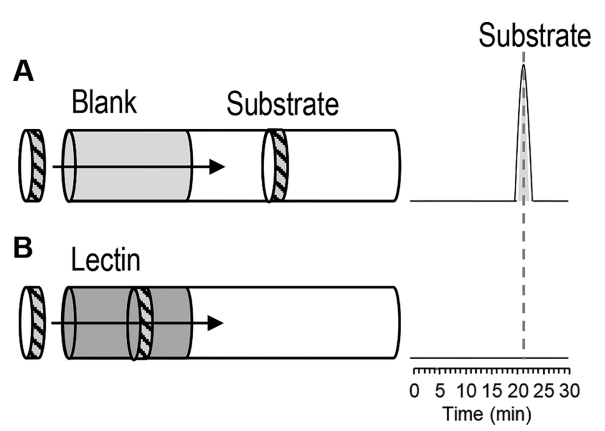

Figure 1. Conceptual diagram illustrating the use of lectins with capillary electrophoresis to identify $\mathrm{N}$-glycan structure. The $\mathrm{N}$-glycan peak appeared in the electropherogram when the capillary contained nanogel (A), but when bound to the lectin, the peak was absent from the trace $(B)$.

selectivity. A stationary plug of lectin was introduced in the capillary as shown in Figure 1. When the structural specificity was a match, the $N$-glycan was bound to the lectin and removed from the electropherogram. The nanogel, which was compatible with the lectin, was made with an aqueous solution that was buffered to a $\mathrm{pH}$ of 6 . This $\mathrm{pH}$ was selected based on the isoelectric points of most lectins, with SNA at $\mathrm{pI}=5$, $^{41}$ $\mathrm{ECL}$ at $\mathrm{pI}=5.0-5.6,{ }^{42}$ PHA-E at $\mathrm{pI}=5.25$ to $6.5,{ }^{43,44}$ and $\mathrm{AAL}$ at $\mathrm{pI}=9 .{ }^{45}$ The performance of other background electrolytes buffered at other $\mathrm{pH}$ values was not evaluated. Separations in the phospholipid nanogel were performed in reversed polarity, because a nanogel coating was used to suppress the electro-osmotic flow. ${ }^{46,47}$ After the capillary was filled with phospholipid, a $0.5 \mathrm{~cm}$ zone of lectin made in phospholipid nanogel was created and moved approximately $2.3 \mathrm{~cm}$ into the capillary. With the commercial instrument used in this work, this positioned the lectin zone near the thermostated region of the separation capillary. Longer zones of lectin could be used with the capillary but were unnecessary. It is possible to retard $\mathrm{N}$-glycans by filling the capillary with lectin rather than concentrating the lectin in a zone (see Figure $\mathrm{S}-2$, traces B and C). However, drawbacks of filling the entire capillary with lectin include the additional flushing required to prevent carryover and the potential for lectin to aggregate or foul the capillary surface at higher concentrations.

Effect of Percent Phospholipid on N-Glycan Separations. The use of nanogel as an additive for capillary electrophoresis had previously been demonstrated with $10 \%$ phospholipid solution with $[\mathrm{DMPC}] /[\mathrm{DHPC}]=2.5 \mathrm{in}$ background electrolyte for the separation of $\mathrm{N}$-glycans derived from alpha-1-acid glycoprotein, fetuin, and RNase B. ${ }^{34}$ The $\mathrm{N}$ glycans all possessed the same core structure. ${ }^{48}$ Human IgG $N$ glycans were predominantly reported to be biantennary and fucosylated ${ }^{15,49}$ and contained varying levels of $\alpha 2-6$-linked sialic acid, galactose, and bisected $N$-acetylglucosamine, ${ }^{49}$ with the most prominent $N$-glycan structures summarized in Figure 2. The $N$-glycans were from a well-characterized commercially available human IgG standard ${ }^{50}$ and separated with $10 \%$ nanogel. As documented in the certificate of analysis, ${ }^{50}$ the IgG standard contained three bisected $\mathrm{N}$-glycans (peaks $4,7,9$ ), but one of these bisected $N$-glycans (peak 7) comigrated with another $\mathrm{N}$-glycan (see Figure 3, upper trace). The comigration
A

B

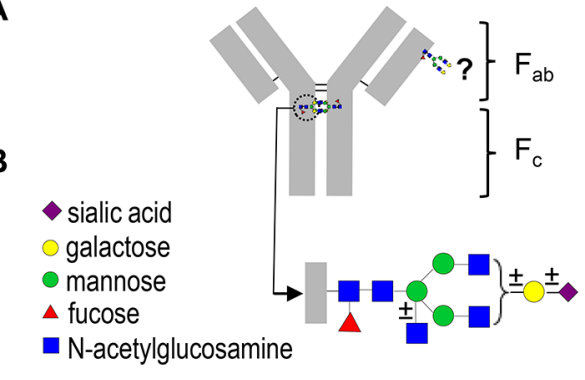

C

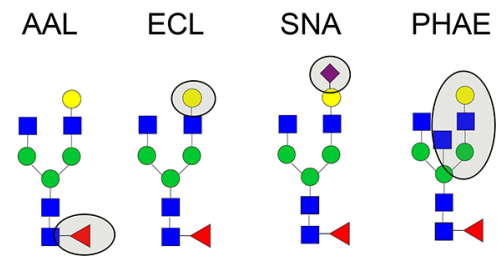

Figure 2. IgG is glycosylated in the $\mathrm{Fc}$ region and possibly in different locations within the Fab region (A). The $\mathrm{N}$-glycans in human IgG are predominantly fucosylated with varying degrees of galactose, $\alpha 2-6$ linked sialic acid, and bisected $N$-acetylglucosamine (B). Fucose, terminal galactose, $\alpha 2-6$-linked sialic acid, and bisected $\mathrm{N}$ acetylglucosamine are recognized by AAL, ECL, SNA, and PHA-E lectin, respectively $(\mathrm{C})$.

of peaks 6 and 7 in capillary electrophoresis separations had been reported by others as well. ${ }^{26,51}$

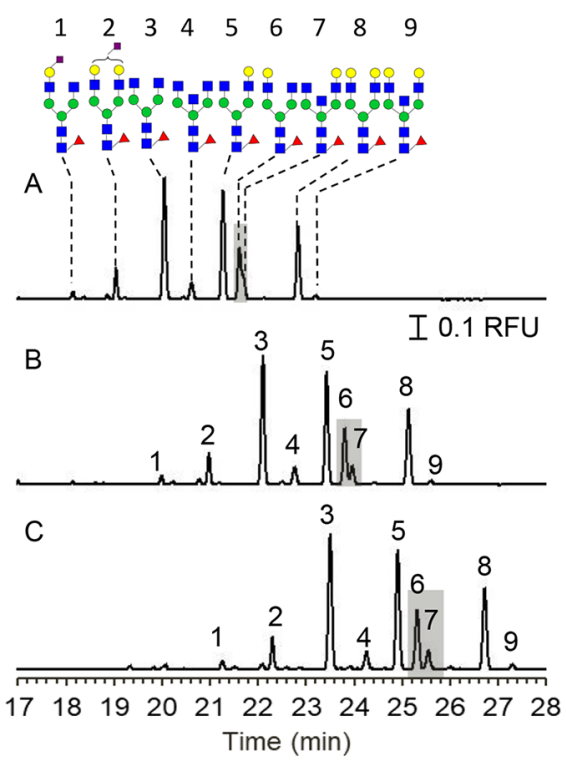

Figure 3. Electropherograms of IgG $N$-glycans obtained with nanogel at $10 \%$ (A), $15 \%$ (B), and $20 \%$ (C). Separations were obtained using a $25 \mu \mathrm{m}$ inner diameter capillary at $17^{\circ} \mathrm{C}$ with an effective length of $52 \mathrm{~cm}$ and a total length of $62 \mathrm{~cm}$. The electric field was $387 \mathrm{~V} / \mathrm{cm}$ in reversed polarity. Sample was injected at $10 \mathrm{kV}$ for $4 \mathrm{~s}$.

The separation enhancement achievable using higher concentrations of nanogel for capillary electrophoresis separations was demonstrated for linear oligosaccharides ${ }^{32}$ but not for $\mathrm{N}$-glycans. As a result, the effect of nanogel concentration on the resolution of these comigrating peaks was investigated at three nanogel concentrations (10, 15, and $20 \%)$. The effect of separation performance was also evaluated for each concentration at a temperature known to generate low 
viscosity $\left(17^{\circ} \mathrm{C}\right)$ and high viscosity $\left(27^{\circ} \mathrm{C}\right)$. The results, summarized in Table S-1, demonstrated that the resolution between peaks 6 and 7 improved with an increase in nanogel concentration, whereas the resolution of peaks 5 and 6 did not change significantly at nanogel concentrations above $10 \%$. In addition, baseline resolution (i.e., resolution above 1.5) was only achieved using $20 \%$ nanogel at $17{ }^{\circ} \mathrm{C}$ for all $\mathrm{N}$-glycan peaks in the IgG sample. This temperature-dependence of the nanogel peak resolution warranted additional experiments to confirm that $17{ }^{\circ} \mathrm{C}$ was the best temperature for the capillary nanogel separation of IgG $\mathrm{N}$-glycans.

In agreement with results reported by others, ${ }^{26,52,53}$ decreasing the separation temperature increased the migration time of all of the $N$-glycan peaks (see Table S-2). A plot of the mobility versus temperature (Figure S-3) revealed nominal change in mobility below $23{ }^{\circ} \mathrm{C}$. At this temperature, nanodiscs were reported as the predominant morphology produced in mixtures of DMPC and DHPC phospholipids. ${ }^{54-56}$ For the separations accomplished using $20 \%$ nanogel, the temperature did not influence the resolution of structural isomers (peaks 5 and 6), but maintaining the separation at $17{ }^{\circ} \mathrm{C}$ improved the resolution of the nonbisected (peak 6) and bisected $N$-glycan (peak 7) that comigrated under other conditions (see Figure 3 and Table S-3). These separations demonstrated that bisected and nonbisected $N$-glycans behaved as two different homologous series, as the hydrodynamic volume and molecular weight were well-correlated (see Figure S-4 and Table S-4), which was previously attributed to the more compact nature of bisected $N$-glycans as compared to non-bisected $N$-glycans. ${ }^{57}$ The best peak resolution was observed at $17{ }^{\circ} \mathrm{C}$, and the temperature did not affect the migration time reproducibility $(\leq 0.08 \%$ relative standard deviation), peak area reproducibility ( $\leq 4 \%$ relative standard deviation), or separation efficiency nor did it result in comigration of the other peaks in the sample (see Table S-5 and Figure S-5). Therefore, the separations were performed using $20 \%$ nanogel at a temperature of $17^{\circ} \mathrm{C}$.

Integration of a Lectin Zone In-Capillary for $\mathrm{N}$-Glycan Characterization. Lectin specificity for different structural features of $\mathrm{N}$-glycans was utilized to confirm the identity of each peak in the electropherogram. The lectin was integrated into the capillary as outlined in Figure 1 , and $N$-glycan structures were revealed by comparing the separations obtained in the absence and the presence of one of four different lectins. When the lectin specificity matches the $\mathrm{N}$ glycan, the peak disappears from the electropherogram, identifying the $\mathrm{N}$-glycan structure. Complex $\mathrm{N}$-glycans have a predictable structure (see Figure 2) with a mannose core at the reducing end. ${ }^{48}$ For IgG $N$-glycans, if fucose is present, it will be at the core ${ }^{49}$ and if sialic acid is present, it will be a terminal residue, and it will be $\alpha 2-6$-linked to a galactose residue. ${ }^{58}$ The nonreducing ends of the mannose cores of the IgG $\mathrm{N}$ glycans contained variable amounts of $\mathrm{N}$-acetylglucosamine, galactose, and $\alpha 2-6$-linked sialic acids and bisected $N$ acetylglucosamine. As a result, peak identification was achieved using only four lectins based on the known $N$-glycan structure. This lectin analysis was accomplished by identifying the presence of fucose on the mannose core with AAL, followed by galactose, $\alpha 2-6$-linked sialic acid, and bisection on the nonreducing end of the $N$-glycan with ECL, SNA, and PHA$\mathrm{E}$, respectively. In these studies, it was not necessary to use the Maackia Amurensis lectin with specificity for $\alpha 2-3$-linked sialic acid, as this linkage is not reported to be present in human
IgG. ${ }^{58}$ The amount of each lectin required was determined by increasing the concentration of lectin in the nanogel zone until no change was observed in the peak area and there was no evidence of retarded $\mathrm{N}$-glycan within the retention window of the major IgG $\mathrm{N}$-glycan peaks (see Figures S-6-9). For runs performed at $17{ }^{\circ} \mathrm{C}$, the relative standard deviation in the area in the absence and in the presence of lectin was at or below 4 and $8 \%$, respectively (see Table S-6).

The stepwise analysis of $N$-glycans with lectins was accomplished as demonstrated in Figure 4. All peaks separated
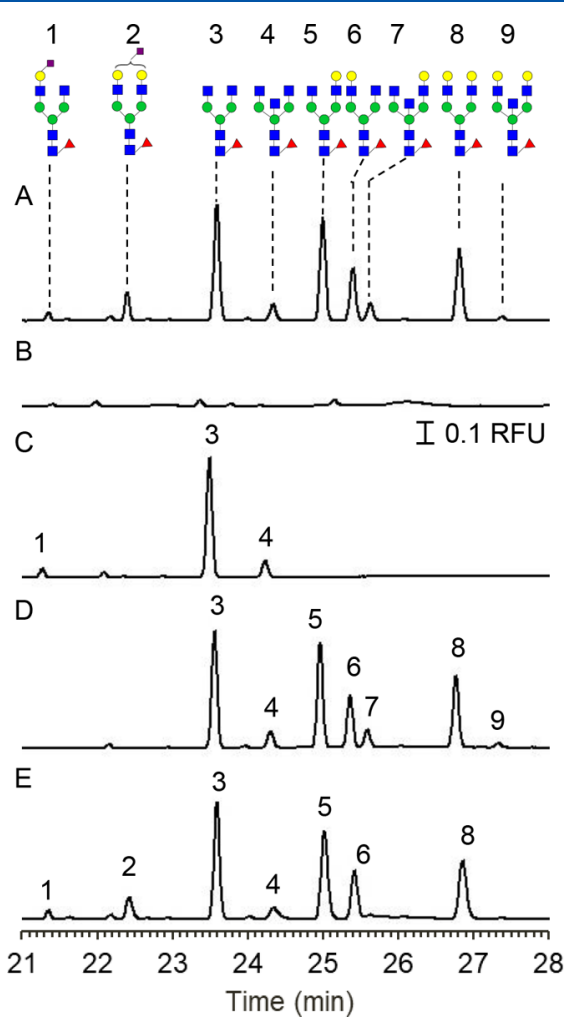

Figure 4. Electropherograms of $\mathrm{IgG}$ glycans were obtained in the absence of lectin (A) or with $100 \mu \mathrm{M}$ AAL selective for fucose (B), $200 \mu \mathrm{M}$ ECL selective for galactose (C), $3 \mu \mathrm{M}$ SNA selective for 2-6linked sialic acid (D), or $5 \mu \mathrm{M}$ PHA-E predominantly selective for bisected glycan but with lower affinity to terminal Gal $\beta 1-4 \mathrm{GlcNAc}$ on $\alpha 1-6$-linked mannose branch (E). Separation conditions were as described in Figure 3.

by the capillary electrophoresis in Figure 4A disappeared with the use of fucose specific lectin (AAL) in Figure 4B, confirming that all of the IgG $\mathrm{N}$-glycans contained fucose. In Figure 4C, galactose specific lectin ECL removed peaks 2, 5, 6, 7, 8, and 9 from the electropherogram, confirming they were galactosylated $\mathrm{N}$-glycans that contained one or two galactose residues. In contrast, peaks 1,3 , and 4 in Figure $4 \mathrm{C}$ indicated the absence of terminal galactose and contained either a terminal $\alpha 2-6$-linked sialic acid (peak 1) or terminal $\mathrm{N}$-acetylglucosamine (peaks 3 and 4). In Figure 4D, a lectin specific for sialic acid containing an $\alpha 2-6$ linkage (SNA) removed two $N$ glycans, confirming that only peaks 1 and 2 contained a terminal $\alpha 2-6$-linked sialic acid. The number of $\alpha 2-6$-linked sialic acid residues present in the biantennary structure of peaks 1 and 2 was elucidated by considering the combined findings from the electropherograms in Figures $4 \mathrm{C}$ and D. Because peak 2 was recognized by both ECL and SNA, this 
peak was attributed to an $\mathrm{N}$-glycan structure containing galactose and $\alpha 2-6$-linked sialic acid at each terminus. Peak 1 , which responded to SNA but not ECL, was attributed to an $\alpha 2-6$-linked sialic acid and $N$-acetylglucosamine at each terminus. Fully sialylated biantennary $N$-glycan was ruled out because of the migration time and the low abundance of the glycoform in human IgG. In a similar manner, peaks 3 and 4 were identified as $\mathrm{N}$-glycans with $\mathrm{N}$-acetylglucosamine at both termini by considering the combined results from the electropherograms obtained in the presence of galactose specific lectin ECL (Figures 4C) and $\alpha 2-6$-linked sialic acid specific lectin (Figure 4D).

Confirmation of Bisected $\mathrm{N}$-Glycan Assignments Based on PHA-E. In Figure 4E, the PHA-E lectin specific for bisected $N$-glycan that also contained a terminal galactose on the $\alpha 1-6$-linked mannose branch ${ }^{31}$ removed peaks 7 and 9, confirming that these $\mathrm{N}$-glycans were bisected. As shown in Figure 4, the PHA-E lectin had a lower affinity for $\mathrm{N}$-glycans containing terminal galactose (i.e., peaks 2, 5, 6, and 8) and for agalactosylated bisected biantennary $N$-glycan (peak 4 ). These peaks had a nominal decrease in peak area (i.e., $\leq 10 \%$ ) but had wider peak widths and greater than a $10 \%$ decrease in the theoretical plate count (see Table S-7 in the Supporting Information). This observed band broadening was used in conjunction with the results of the galactose-selective ECL lectin separation in Figure $4 \mathrm{C}$ to confirm that peak 4 was the agalactosylated bisected biantennary $\mathrm{N}$-glycan. To demonstrate the selectivity of PHA-E lectin, two bisected N-glycan standards were separated in the presence of this lectin and verified the interactions observed for the IgG $\mathrm{N}$-glycans in Figure 4. The agalactosylated bisected $N$-glycan standard corresponding to peak 4 was observed to decrease in plate count but remained in the electropherogram, whereas the bigalactosylated bisected $N$-glycan standard corresponding to peak 9 was removed from the electropherogram (see Figure S$10)$.

Identification of $\mathbf{N}$-Glycans of Minor Abundance. A total of nine abundant $N$-glycans (i.e., peaks 1-9) are identified with four lectins; yet other small peaks are observed in the electropherogram. Some residual peaks in the electropherogram are contaminants; however, six additional minor peaks are identified as $N$-glycans as well (Figure S-11 and Table S-8). These six $N$-glycans constitute approximately $4 \%$ of the sum of the areas associated with $\mathrm{N}$-glycans attributed to peaks 1 through 9. A seventh peak can be identified if Griffonia simplicifolia lectin II is used, which is a lectin specific for $\beta$ linked $\mathrm{N}$-acetylglucosamine residues (Figure $\mathrm{S}-12$ ). The integration of lectin and capillary nanogel electrophoresis provides a means to distinguish contaminant peaks that may be confused for $\mathrm{N}$-glycans, offering an additional means of structural identification.

Profiling IgG $\mathbf{N}$-Glycans from Different IgG Samples. Following the identification of the major IgG $N$-glycans using online lectin analyses (i.e., peaks 1 through 9), capillary nanogel electrophoresis provided a quantitative comparison between two different IgG samples. The $N$-glycans from different IgG samples were introduced in the separation capillary with a hydrodynamic injection to prevent preferential injection observed with electrokinetic injections arising from differences in the sample matrix. No difference in migration time of the $\mathrm{N}$-glycan peaks was observed between electrokinetic and hydrodynamic injections. The relative abundance of each $\mathrm{N}$-glycan in these different samples was compared by normalizing the samples to the signal for the agalactosylated bisected $N$-glycan (i.e., peak 4). This was accomplished by adjusting the concentration of each sample to the same peak area for the agalactosylated bisected $\mathrm{N}$-glycan (peak 4). The solid trace in Figure 5 was the same IgG sample shown in

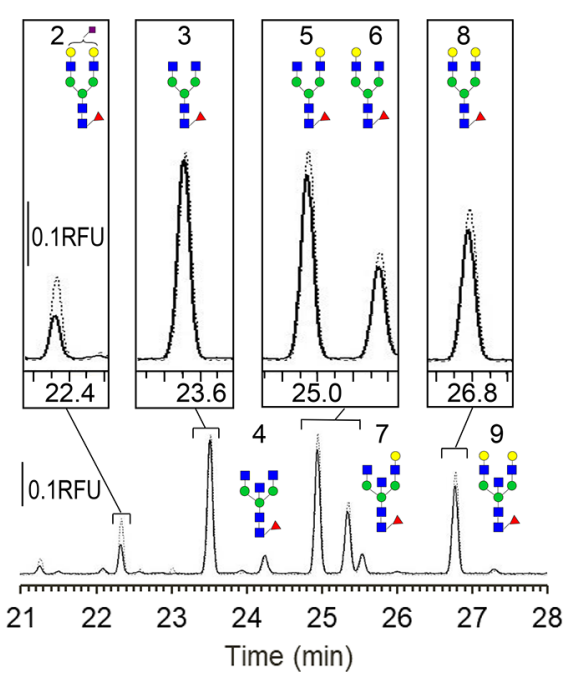

Figure 5. Overlaid electropherograms to profile IgG $N$-glycans. The solid trace is of the sample depicted in Figures 3 and 4, and the dotted trace is of $\mathrm{N}$-glycans derived from a different source of IgG. The concentration of the samples was adjusted to make peak 4 the same area in order to compare relative abundance. Separation conditions were as described in Figure 3, with the exception of sample injection at $3 \mathrm{psi}$ for $5 \mathrm{~s}$.

Figures 3 and 4, whereas the dotted trace contained $N$-glycans derived from the new IgG sample. Notably, all of the major $N$ glycans were fucosylated. The peak areas of the bisected $\mathrm{N}$ glycans (peaks 4 and 7) were not significantly different (see Table 1). The total amount of bisected $N$-glycan in samples 1 and 2 was 9.1 and $7.5 \%$, respectively (see the electropherograms in Figure S-13 and Table S-9). Conversely, most of the peak areas of the non-bisected $\mathrm{N}$-glycans, except for peak 3, were significantly different, with sample 1 containing higher

Table 1. Comparison of Glycan Profiles ${ }^{a}$

\begin{tabular}{|c|c|c|c|c|}
\hline \multirow[b]{2}{*}{ peak } & \multirow{2}{*}{$\begin{array}{c}\text { sample } 1 \\
\text { area } \times 10^{5} \\
\bar{X}(\% \text { RSD })\end{array}$} & \multirow{2}{*}{$\begin{array}{c}\text { sample } 2 \\
\text { area } \times 10^{5} \\
\bar{X}(\% \mathrm{RSD})\end{array}$} & \multicolumn{2}{|c|}{ difference } \\
\hline & & & absolute & (relative) \\
\hline 1 (G1FS1) & 0.08 (4) & $0.17(2)$ & 0.08 & $(67 \%)^{b}$ \\
\hline 2 (G2FS1) & 0.29 (3) & $0.54(0.6)$ & 0.25 & $(59 \%)^{b}$ \\
\hline 3 (G0F) & $1.62(4)$ & $1.66(0.7)$ & 0.03 & $(2 \%)$ \\
\hline 4 (G0FB) & $0.23(4)$ & $0.22(0.8)$ & 0.01 & $(3 \%)$ \\
\hline $5(\mathrm{G} 1 \mathrm{~F})$ & $1.52(4)$ & $1.68(0.4)$ & 0.17 & $(10 \%)^{b}$ \\
\hline $6(\mathrm{G} 1 \mathrm{~F})$ & $0.80(4)$ & $0.91(0.4)$ & 0.11 & $(13 \%)^{b}$ \\
\hline 7 (G1FB) & 0.25 (4) & $0.24(0.9)$ & 0.01 & $(6 \%)$ \\
\hline 8 (G2F) & $1.14(4)$ & $1.31(0.3)$ & 0.17 & $(14 \%)^{b}$ \\
\hline 9 (G2FB) & $0.06(3)$ & $0.05(3)$ & 0.01 & $(20 \%)^{b}$ \\
\hline
\end{tabular}

${ }^{a}$ Average and coefficient of variation determined from triplicate runs. Sample 1 is Glyko $^{\circledR}$ APTS Human IgG N-Linked Glycan Library (Prozyme). Sample 2 is glycan cleaved from human $\operatorname{IgG}$ (Lot \# SLBK8678V, Sigma-Aldrich). Relative differences between samples calculated as $\left(\right.$ area $_{\text {sample1 }}-$ area $\left._{\text {sample } 2}\right) /$ average area. ${ }^{b}$ Values have significantly different areas as calculated using a $t$ test with $95 \%$ confidence. 
amounts of these non-bisected $N$-glycans than sample 2 (see Table 1 and Figure 5). The percentages of non-bisected $\mathrm{N}$ glycans that contained $\alpha 2-6$-linked sialic acid (peaks 1 and 2) relative to the total $\mathrm{N}$-glycan composition were only 6.3 and $10.4 \%$ in samples 1 and 2, respectively. The peak areas of the non-bisected $N$-glycans that lacked $\alpha 2-6$-linked sialic acid (peaks 3, 5, 6, and 8) comprised over $80 \%$ of the total $N$-glycan composition for both samples. The distributions of biantennary $N$-glycans (see Table S-9) containing 0 (G0F), 1 (G1F), or 2 (G2F) terminal galactose residues for samples 1 and 2 were similar to 27 and $24 \% \mathrm{GOF}, 39$ and $38 \% \mathrm{G} 1 \mathrm{~F}$, and 19 and $19 \%$ G2F, respectively.

The commercial standards selected for this study were useful to demonstrate the power of the method to evaluate the $\mathrm{N}$ glycan profile but were intended for use as a chemical standard rather than a clinical standard. Although the effectiveness of capillary electrophoresis for profiling $N$-glycans was demonstrated in Figure 5 and Table 1, several factors made it difficult to draw global conclusions from these results based on published literature values of IgG $\mathrm{N}$-glycan heterogeneity. Glycosylation levels vary considerably in humans. ${ }^{4,59}$ In addition, analytical studies designed to profile IgG $N$-glycans were confounded by differences in the distributions of $\mathrm{N}$ glycans at the $\mathrm{Fab}$ and $\mathrm{Fc}$ regions of the antibody, ${ }^{60,61}$ which required enzymatic treatment designed to cleave the Fab and Fc regions of the IgG antibody ${ }^{62}$ or treatment with PNGase F performed without denaturing the protein. ${ }^{60,61}$ With these caveats, it was noted that the relative abundance of bisecting $\mathrm{N}$-acetylglucosamine was similar to some reports in the literature. ${ }^{16,63}$ Furthermore, the abundance of the non-bisected galactosylated $\mathrm{N}$-glycans was similar to a report that approximated the amount of $\mathrm{N}$-glycans containing zero (G0F), one (G1F), or two (G2F) terminal galactose residues derived from commercially available human $\operatorname{IgG}$ at 25,33 , and $15 \%$, respectively. ${ }^{63}$ The same authors reported the amount of $\mathrm{N}$-glycans containing zero (G0F), one (G1F), or two (G2F) terminal galactose residues from IgG derived from healthy humans at 21,38 , and $16 \%$, respectively. ${ }^{63}$ There was a notable difference in the relative abundance of sialylated $\mathrm{N}$-glycans (i.e., 6.3 and $10.4 \%$ for samples 1 and 2, respectively) from both samples as compared to literature values of 12 to $25 \%$ reported by others. ${ }^{16,23,63,64}$ In this study, no effort was made to prevent loss of sialic acid during the deglycosylation step, in which protein used to derive sample $2 \mathrm{~N}$-glycans was denatured at $80{ }^{\circ} \mathrm{C}$ for $2 \mathrm{~min}$. Hydrolysis of sialic acids, for example at elevated temperatures, would decrease the amount of sialylated $\mathrm{N}$-glycan observed and increase the asialylated $\mathrm{N}$ glycans (i.e., peaks 5, 6, and 8). The results in the literature point to the need for a low-cost, automated, and accessible method to profile IgG $\mathrm{N}$-glycans and the potential of the capillary electrophoresis as enabling technology to complement other approaches for $N$-glycan identification.

\section{CONCLUSIONS AND FUTURE DIRECTIONS}

The applicability of nanogel electrophoresis to $N$-glycan analysis was expanded to include the use of lectins to identify $\mathrm{N}$-glycan composition without the need for $\mathrm{N}$-glycan standards. In conjunction with a series of four lectins (AAL, ECL, SNA, and PHA-E), biantennary $N$-glycans derived from human IgG protein were identified. The conclusive detection of all bisected $\mathrm{N}$-glycans was not been previously demonstrated with PHA-E. The lower affinity of the PHA-E lectin for N-glycan that contained terminal galactose in the absence of bisected $\mathrm{N}$ - acetylglucosamine as well as for $\mathrm{N}$-glycans that were agalactosylated bisected biantennary was leveraged when lectins were integrated in capillary electrophoresis, because the lower affinity to these motifs was observed in the electropherograms as a change in the peak width. This change in width, when used in conjunction with the results obtained with the ECL lectin, enabled the assignment of the agalactosylated bisected biantennary $N$-glycan. The profiles from two different IgG samples revealed significant differences in the amount of non-bisected $N$-glycans, except for peak 3 . When the two samples were normalized to the concentration of the agalactosylated bisected biantennary $N$-glycan, no significant difference in the distribution of bisected $\mathrm{N}$-glycans was observed. Analyses of $\mathrm{N}$-glycans were achieved with separation efficiencies of approximately 500000 theoretical plates using $20 \% \mathrm{w} / \mathrm{v}$ nanogel. Although the change in temperature did not impact efficiency, it enabled the resolution of monogalactosylated biantennary $N$-glycan from bisected monogalactosylated biantennary $N$-glycan. This role of temperature in resolving bisected and non-bisected $\mathrm{N}$-glycans had previously been observed only with linear polymers used as a separation additive but has now been demonstrated in selfassembled nanogels.

The nanogel electrophoresis separations that incorporate lectins are rapid, automated, and cost-effective. This method holds potential to rapidly screen IgG samples for the degree of bisected $\mathrm{N}$-glycan, which is relevant to the therapeutic efficacy of IgG monoclonal antibody pharmaceuticals. In addition, the method is significant to the rapid identification of bisection in IgG from human serum and complements existing technologies used to shed light on the prognostic and diagnostic relevance of this motif.

\section{ASSOCIATED CONTENT}

\section{S Supporting Information}

The Supporting Information is available free of charge on the ACS Publications website at DOI: 10.1021/acs.analchem.8b03725.

List of chemicals, effects of nanogel concentration and temperature on the separation resolution of IgG $\mathrm{N}$ glycans, effects of temperature on migration time, mobility, and plate count, relationship of mobility and molecular weight, concentration study of each lectin, reproducibility in area and plate count in the presence of lectin, expanded electropherogram of IgG N-glycan profiles (major and minor peaks), percent of peak areas, and the migration times of the $N$-glycan peaks (PDF)

\section{AUTHOR INFORMATION}

\section{Corresponding Author}

*E-mail: Lisa.Holland@mail.wvu.edu.

\section{ORCID}

Lisa A. Holland: 0000-0002-7534-6810

\section{Notes}

The authors declare no competing financial interest.

\section{ACKNOWLEDGMENTS}

This material is based upon work supported by NIH Grant No. R01GM114330. 


\section{REFERENCES}

(1) Krapp, S.; Mimura, Y.; Jefferis, R.; Huber, R.; Sondermann, P. J. Mol. Biol. 2003, 325, 979-989.

(2) Gabius, H.-J. BioSystems 2018, 164, 102-111.

(3) Kailemia, M. J.; Park, D.; Lebrilla, C. B. Anal. Bioanal. Chem. 2017, 409, 395-410.

(4) Adamczyk, B.; Tharmalingam, T.; Rudd, P. M. Biochim. Biophys. Acta, Gen. Subj. 2012, 1820, 1347-1353.

(5) Kodar, K.; Stadlmann, J.; Klaamas, K.; Sergeyev, B.; Kurtenkov, O. Glycoconjugate J. 2012, 29, 57-66.

(6) Theodoratou, E.; Thaçi, K.; Agakov, F.; Timofeeva, M. N.; Stambuk, J.; Pučić-Baković, M.; Vučković, F.; Orchard, P.; Agakova, A.; Din, F. V. N.; et al. Sci. Rep. 2016, 6, 28098.

(7) Alley, W. R.; Vasseur, J. A.; Goetz, J. A.; Svoboda, M.; Mann, B. F.; Matei, D. E.; Menning, N.; Hussein, A.; Mechref, Y.; Novotny, M. V. J. Proteome Res. 2012, 11, 2282-2300.

(8) Chen, J.; Fang, M.; Zhao, Y.-P.; Yi, C.-H.; Ji, J.; Cheng, C.; Wang, M.-M.; Gu, X.; Sun, Q.-S.; Chen, X.-L.; Gao, C.-F. PLoS One 2015, 10, e0127022.

(9) Reiding, K. R.; Vreeker, G. C. M.; Bondt, A.; Bladergroen, M. R.; Hazes, J. M. W.; van der Burgt, Y. E. M.; Wuhrer, M.; Dolhain, R. J. E. M. Front. Med. 2018, 4, 241.

(10) Trbojević Akmačíc, I.; Ventham, N. T.; Theodoratou, E.; Vučković, F.; Kennedy, N. A.; Krištić, J.; Nimmo, E. R.; Kalla, R.; Drummond, H.; Štambuk, J.; et al. Inflammatory Bowel Dis 2015, 21, 1237-1247.

(11) Jefferis, R. Curr. Pharm. Biotechnol. 2016, 17, 1333-1347.

(12) Vidarsson, G.; Dekkers, G.; Rispens, T. Front. Immunol. 2014, 5,520 .

(13) Raju, T. S.; Briggs, J. B.; Borge, S. M.; Jones, A. J. S. Glycobiology 2000, 10, 477-486.

(14) Pučić, M.; Knežević, A.; Vidič, J.; Adamczyk, B.; Novokmet, M.; Polašek, O.; Gornik, O.; Supraha-Goreta, S.; Wormald, M. R.; Redžić, I.; et al. Cell. Proteomics 2011, 10, M111.010090.

(15) Parekh, R. B.; Dwek, R. A.; Sutton, B. J.; Fernandes, D. L.; Leung, A.; Stanworth, D.; Rademacher, T. W.; Mizuochi, T.; Taniguchi, T.; Matsuta, K.; Takeuchi, F.; Nagano, Y.; Miyamoto, T.; Kobata, A. Nature 1985, 316, 452-457.

(16) Bondt, A.; Rombouts, Y.; Selman, M. H. J.; Hensbergen, P. J.; Reiding, K. R.; Hazes, J. M. W.; Dolhain, R. J. E. M.; Wuhrer, M. Mol. Cell. Proteomics 2014, 13, 3029-3039.

(17) Kobata, A. Biochim. Biophys. Acta, Gen. Subj. 2008, 1780, 472478.

(18) Takahashi, M.; Kuroki, Y.; Ohtsubo, K.; Taniguchi, N. Carbohydr. Res. 2009, 344, 1387-1390.

(19) Ferrara, C.; Brünker, P.; Suter, T.; Moser, S.; Püntener, U.; Umaña, P. Biotechnol. Bioeng. 2006, 93, 851-861.

(20) Castilho, A.; Gattinger, P.; Grass, J.; Jez, J.; Pabst, M.; Altmann, F.; Gorfer, M.; Strasser, R.; Steinkellner, H. Glycobiology 2011, 21, 813-823.

(21) Hendrickson, O. D.; Zherdev, A. V. Crit. Rev. Anal. Chem. 2018, 48, 279-292.

(22) Zhang, L.; Luo, S.; Zhang, B. $m A$ ss 2016, 8, 524-535.

(23) Adamczyk, B.; Tharmalingam-Jaikaran, T.; Schomberg, M.; Szekrényes, Á.; Kelly, R. M.; Karlsson, N. G.; Guttman, A.; Rudd, P. M. Carbohydr. Res. 2014, 389, 174-185.

(24) Kawai, T.; Ota, N.; Imasato, A.; Shirasaki, Y.; Otsuka, K.; Tanaka, Y. J. Chromatogr. A 2018, 1565, 138-144.

(25) Jarvas, G.; Szigeti, M.; Chapman, J.; Guttman, A. Anal. Chem. 2016, 88, 11364-11367.

(26) Guttman, A.; Kerekgyarto, M.; Jarvas, G. Anal. Chem. 2015, 87, $11630-11634$.

(27) Lu, G.; Crihfield, C. L.; Gattu, S.; Veltri, L. M.; Holland, L. A. Chem. Rev. 2018, 118, 7867-7885.

(28) Yamamoto, S.; Shinohara, C.; Fukushima, E.; Kakehi, K.; Hayakawa, T.; Suzuki, S. J. Chromatogr. A 2011, 1218, 4772-4778.

(29) Yagi, Y.; Yamamoto, S.; Kakehi, K.; Hayakawa, T.; Ohyama, Y.; Suzuki, S. Electrophoresis 2011, 32, 2979-2985.
(30) Kinoshita, M.; Kakehi, K. Methods Mol. Biol. (N. Y., NY, U. S.) 2014, 1200, 131-146.

(31) Yamashita, K.; Hitoi, A.; Kobata, A. J. Biol. Chem. 1983, 258, $14753-14755$.

(32) Wu, X.; Langan, T. J.; Durney, B. C.; Holland, L. A. Electrophoresis 2012, 33, 2674-2681.

(33) Pappas, T.; Holland, L. Sens. Actuators, B 2008, 128, 427-434.

(34) Luo, R.; Archer-Hartmann, S. A.; Holland, L. A. Anal. Chem. 2010, 82, 1228-1233.

(35) Gattu, S.; Crihfield, C. L.; Holland, L. A. Anal. Chem. 2017, 89, 929-936.

(36) Holland, L. A.; Gattu, S.; Crihfield, C. L.; Bwanali, L. J. Chromatogr. A 2017, 1523, 90-96.

(37) Archer-Hartmann, S. A.; Sargent, L. M.; Lowry, D. T.; Holland, L. A. Anal. Chem. 2011, 83, 2740-2747.

(38) Archer-Hartmann, S. A.; Crihfield, C. L.; Holland, L. A. Electrophoresis 2011, 32, 3491-3498.

(39) New England Biolabs. Rapid PNGase F Protocols (P0710). https://www.neb.com/protocols/2014/10/23/rapid-phgase-fprotocols-f-p0710, accessed August 1, 2018.

(40) Váradi, C.; Lew, C.; Guttman, A. Anal. Chem. 2014, 86, 56825687.

(41) Emig, S.; Schmalz, D.; Shakibaei, M.; Buchner, K. J. Biol. Chem. 1995, 270, 13787-13793.

(42) Bhattacharyya, L.; Haraldsson, M.; Sharon, N.; Lis, H.; Brewer, F. Glycoconjugate J. 1989, 6, 141-150.

(43) Miller, J. B.; Noyes, C.; Heinrikson, R.; Kingdon, H. S.; Yachnin, S. J. Exp. Med. 1973, 138, 939-951.

(44) Weber, T. H.; Aro, H.; Nordman, C. T. Biochim. Biophys. Acta, Protein Struct. 1972, 263, 94-105.

(45) Matsumura, K.; Higashida, K.; Ishida, H.; Hata, Y.; Yamamoto, K.; Shigeta, M.; Mizuno-Horikawa, Y.; Wang, X.; Miyoshi, E.; Gu, J.; Taniguchi, N. J. Biol. Chem. 2007, 282, 15700-15708.

(46) Cunliffe, J. M.; Baryla, N. E.; Lucy, C. A. Anal. Chem. 2002, 74, $776-783$.

(47) White, C. M.; Luo, R.; Archer-Hartmann, S. A.; Holland, L. A. Electrophoresis 2007, 28, 3049-3055.

(48) Stanley, P.; Schachter, H.; Taniguchi, N. N-Glycans. In Essentials of Glycobiology, 2nd ed.; Varki, A., Cummings, R. D., Esko, J. D., Freeze, H. H., Stanley, P., Bertozzi, C. R., Hart, G. W., Etzler, M. E., Eds.; Cold Spring Harbor Laboratory Press: Cold Spring Harbor, NY, 2009.

(49) Zauner, G.; Selman, M. H. J.; Bondt, A.; Rombouts, Y.; Blank, D.; Deelder, A. M.; Wuhrer, M. Mol. Cell. Proteomics 2013, 12, 856865.

(50) Prozyme. Glyko® APTS Human IgG N-Linked Glycan Library [GKSP-005], Lot Number P14A2001b. https://prozyme.com/ products/gksp-005, accessed November 27, 2018.

(51) Mittermayr, S.; Bones, J.; Doherty, M.; Guttman, A. s.; Rudd, P. M. J. Proteome Res. 2011, 10, 3820-3829.

(52) Jarvas, G.; Kerekgyarto, M.; Guttman, A. Electrophoresis 2016, 37, 2347-2351.

(53) Kerékgyártó, M.; Járvás, G.; Novák, L.; Guttman, A. Electrophoresis 2016, 37, 573-578.

(54) Sanders, C. R., II; Schwonek, J. P. Biochemistry 1992, 31, $8898-8905$

(55) Nieh, M. P.; Raghunathan, V. A.; Glinka, C. J.; Harroun, T. A.; Pabst, G.; Katsaras, J. Langmuir 2004, 20, 7893-7897.

(56) Rowe, B. A.; Neal, S. L. Langmuir 2003, 19, 2039-2048.

(57) Mitra, I.; Snyder, C. M.; Zhou, X.; Campos, M. I.; Alley, W. R.; Novotny, M. V.; Jacobson, S. C. Anal. Chem. 2016, 88, 8965-8971.

(58) Takahashi, N.; Nakagawa, H.; Fujikawa, K.; Kawamura, Y.; Tomiya, N. Anal. Biochem. 1995, 226, 139-146.

(59) Huhn, C.; Selman, M. H. J.; Ruhaak, L. R.; Deelder, A. M.; Wuhrer, M. Proteomics 2009, 9, 882-913.

(60) Anumula, K. R. J. Immunol. Methods 2012, 382, 167-176.

(61) Mimura, Y.; Ashton, P. R.; Takahashi, N.; Harvey, D. J.; Jefferis, R. J. Immunol. Methods 2007, 326, 116-126. 
(62) von Pawel-Rammingen, U.; Johansson, B. P.; Björck, L. EMBO J. 2002, 21, 1607-1615.

(63) Flynn, G. C.; Chen, X.; Liu, Y. D.; Shah, B.; Zhang, Z. Mol. Immunol. 2010, 47, 2074-2082.

(64) Mizuochi, T.; Taniguchi, T.; Shimizu, A.; Kobata, A. J. Immunol. 1982, 129, 2016-2020. 